\title{
Influência da temperatura sobre a composição físico-química de noni desidratado (Morinda citrifolia L.)
}

\author{
Victor Herbert de Alcântara Ribeiro ${ }^{1}$; Sâmela Leal Barros ${ }^{2}$; Newton Carlos Santos ${ }^{3}$; Virgínia Mirtes de Alcântara \\ Silva ${ }^{4} ;$ Mylena Olga Pessoa Melo ${ }^{5}$; Amanda Priscila Silva Nascimento ${ }^{6}$

\begin{abstract}
Universidade Federal de Campina Grande, ${ }^{1}$ victor_herbert@gmail.com, ${ }^{2}$ samelaleal7 @gmail.com, 33ewtonquimicoindustrial@gmail.com, ${ }^{4}$ virginia.mirtes2015@gmail.com, ${ }^{5}$ mylenaopm@gmail.com, 6amandapriscil@yahoo.com.br.
\end{abstract}

\begin{abstract}
RESUMO: O noni é uma fruta exótica e pouco estudada no Brasil, porém há pesquisas que apontam seu elevado valor nutricional associado ao seu potencial antioxidante. Objetivou-se por meio do presente estudo, avaliar o potencial biotecnológico do noni, através da caracterização físico-química da fruta in natura e desidratada através de secagem convectiva, que foi realizada em secador com circulação de ar com as temperaturas de 50,60 e $70{ }^{\circ} \mathrm{C}$. As amostras in naturae desidratadas foram avaliadas com relação aos seguintes parâmetros: umidade, atividade de água, $\mathrm{pH}$, acidez, teor de sólidos solúveis totais, ratio, proteínas, cinzas, vitamina $\mathrm{C}$ e lipídeos. Foi observado que o aumento da temperatura proporcionou a redução do teor de umidade e de atividade de água do produto, influenciando positivamente na estabilidade do produto. De maneira oposta, foi observada o aumento da acidez, sólidos solúveis totais, lipídeos, cinzas e vitamina $\mathrm{C}$.
\end{abstract}

PALAVRAS-CHAVE: Fitoterápico; Qualidade; Secagem; Vitamina C.

\section{INTRODUÇÃO}

O noni (Morinda citrifolia L.) é uma fruta proveniente do sudoeste da Ásia, que possui coloração da casca branca eformatos que variam de diformes a ovalados. Durante o estádio maduro de maturação, a polpa apresenta textura mole e quebradiça, da qual é desprendido um forte odor que é provavelmente originado de ácidos orgânicos, dentre os quais destacam-se os ácidos octanoico e etanoico. $\mathrm{O}$ alto valor nutricional associado às propriedades fitoterápicas e a grande facilidade de adaptação são os principais fatores responsáveis pela expansão do cultivo do noni em diversas partes do mundo e em regiões brasileiras (SOUSA et al., 2017).

As propriedades fitoterápicas associadas ao noni são provenientes de aproximadamente 200 substâncias bioativas, como os compostos fenólicos, alcaloides e ácidos orgânicos que possuem ações anti-inflamatórias, antioxidantes e anti-helmínticas. Além de impulsionar positivamente o sistema imunológico, contribuindo para a maior produção de macrófagos e linfócitos, atuando nas defesas do organismo e auxiliando no combate a bactérias, vírus e células cancerígenas. Também são verificados contribuições no combate a infecção, artrite, hipertensão, asma e diabetes. No entanto, ainda existem poucos estudos para evidenciar essas propriedades funcionais e nutricionais (LIMA et al., 2017; LIMA et al., 2018).

O consumo do fruto da Morinda citrifolia $L$ é frequente na Ásia onde o fruto é extremamente popular, porém no Brasil o cultivo do fruto foi iniciado recentemente e ainda não há muitas informações relacionadas as propriedades nutricionais e toxicológicas do produto. Apesar dos diversos estudos existentes com relação ao noni, a Agência Nacional de Vigilância Sanitária (ANVISA), limitou por meio da Resolução RDC nº. 278/2005 a comercialização de qualquer alimento contendo a fruta, por considerar o histórico de consumo no Brasil insuficiente para determinar se a ingestão da fruta pode ou não causar efeitos adversos à saúde humana. Então, para que o produto possa ser comercializado, as empresas devem comprovar a segurança no consumo do noni e solicitar o registro do produto na ANVISA (PIMENTEL et al., 2016).

No Brasil, a difusão do suco da fruta de noni ainda é muito recente, não existindo relatos de produção em escala comercial dessa fruta no país. Existe a necessidade de comprovar as propriedades nutracêuticas do noni, assim como determinar a melhor forma de processamento para este fruto, bem como a melhor maneira de utilizar seus produtos a fim de manter suas propriedades benéficas e aumentar a sua aceitação sensorial (SANDES et al., 2018).

$\mathrm{O}$ processo de secagem proporciona a redução do teor de água do produto, aumentando significativamente sua vida útil, influenciando também na palatabilidade e digestibilidade. Além disso, proporciona o desenvolvimento de novos produtos, com redução de custos operacionais. Contudo, a seleção das condições adequadas para a operação de secagem devem ser efetuadas através de analises físico-químicas que permitem a observação da influência do binômio (tempo x temperatura) sobre a qualidade nutricional do produto (FIGUEIREDO et al., 2017).

Diante do exposto, o presente estudo tem como objetivo investigar o potencial biotecnológico do noni, através da caracterização físico-química da fruta in natura e desidratada através de secagem convectiva. 
RIBEIRO, V. H. A et al. Influência da temperatura sobre a composição físico-química de noni desidratado (Morinda citrifolia L.). In: II Congresso Paraibano de Agroecologia \& IV Exposição Tecnológica, 2019. Anais... Caderno Verde de Agroecologia e

Desenvolvimento Sustentável, Pombal, v. 9, n.7, e-7099, 2019.

\section{MATERIAL E MÉTODOS}

\section{Obtenção das amostras}

Os nonis (Morinda citrifolia L.) foram adquiridas em uma feira central localizada na cidade de Campina Grande, Paraaíba, em seguida foram transportadas para posterior análise. As análises foram realizadas no Laboratório de Secagem, pertencente à Unidade Acadêmica de Engenharia Alimentos, da Universidade Federal de Campina Grande, PB.

\section{Desidratação dos frutos}

Os frutos foram selecionados, lavados e higienizados. Após remoção das sementes a polpa do noni foi colocada em bandejas de aço inoxidável, e uniformemente espalhadas, formando uma camada fina. Para iniciar o experimento, utilizando as temperaturas de 60,70 e $80^{\circ} \mathrm{C}$ até atingirem o equilíbrio higroscópico. No final das secagens foram determinadas as massas secas e calculados os teores de umidade final.

\section{Análises físico-químicas}

As amostras in natura e desidratadas foram caracterizadas com relação aos seguintes parâmetros físicoquímicos: por secagem em estufa a $105{ }^{\circ} \mathrm{C}$ até peso constante, o teor de cinzas foi determinado por incineração em mufla; $\mathrm{pH}$ foi obtido através de leitura direta no medidor de $\mathrm{pH}$ digital, a acidez total e o teor de ácido ascórbico (vitamina C) determinadapor titulometria, de acordo com as metodologias propostas pelo Instituto Adolfo Lutz (IAL, 2008).O teor de proteína total foi quantificado pelo método de Micro-Kjeldahl, que consistiu na determinação do nitrogênio total, O teor de lipídeos foi determinado pelo método modificado de Blig e Dyer (1959); O teor total de carboidratos foi calculado por diferença para obter $100 \%$ da composição total (FAO, 2003). A atividade de água ( $\left.\mathrm{a}_{\mathrm{w}}\right)$ foi determinada usando o dispositivo Decagon ${ }^{\circledR}$ Aqualab CX-2T a $25^{\circ} \mathrm{C}$.

\section{Análise estatística}

Os dados obtidos com relação a caracterização físico-química das amostras desidratadas foram avaliados estatisticamente, por meio de um delineamento inteiramente casualizado, e as medias comparadas pelo teste de Tukey a 5\% de probabilidade, com o auxílio do programa estatistico ASSISTAT versao 7.7 beta (SILVA e AZEVEDO, 2016).

\section{RESULTADOS E DISCUSSÃO}

Na Tabela 1 estão expressos os resultados referentes a caracterização da polpa de noni in natura.

Tabela 1- Caracterização físico-química da polpa de noni in natura

\begin{tabular}{cc}
\hline Parâmetros & Polpa de noni in natura \\
\hline Umidade $(\%)$ & $92,42 \pm 0,03$ \\
Atividade de água $\left(\mathrm{A}_{\mathrm{w}}\right)$ & $0,940 \pm 0,01$ \\
$\mathrm{pH}$ & $4,38 \pm 0,02$ \\
ATT (\% ácido cítrico) & $0,55 \pm 0,05$ \\
SST ( $\left.{ }^{(} \mathrm{Brix}\right)$ & $10,10 \pm 0,01$ \\
Ratio $(\mathrm{SST} / \mathrm{ATT})$ & $18,36 \pm 0,001$ \\
Vitamina C (mg/100g) & $154,2 \pm 0,12$ \\
Cinzas $(\%)$ & $0,72 \pm 0,23$ \\
Proteínas (\%) & $1,15 \pm 0,15$ \\
Lipídeos $(\%)$ & $2,15 \pm 0,11$ \\
\hline
\end{tabular}

Através dos resultados expressos na Tabela 1, observa-se que o noni in natura apresenta elevado teor de umidade $(92,42 \%)$ e de atividade de água $(0,940)$. Estes valores são superiores ao obtido por Silva et al. (2019) em polpa de Cereus jamacaru para o teor de umidade $(86,23 \%)$ e inferior com relação ao teor de água $(0,99 \%)$.

O noni apresenta elevada acidez, fato observado através dos parâmetros pH $(4,38)$ e acidez $(0,55 \%)$. Estes valores quando comparados com o obtido para pitaia-rosa, estudada por Cordeiro et al. (2015) que apresentou pH de 5,38 e acidez de $0,29 \%$, indica que o noni apresenta maior acidez.

Com relação ao teor de sólidos solúveis, a amostra apresentou $10,10^{\circ}$ Brix, valor superior ao obtido por Faria et al. (2014) em noni cultivado na cidade de Cuiabá ( $9,2{ }^{\circ}$ Brix).A relação entre o teor de sólidos solúveis (ratio) foi de 18,36. De acordo com Morgado et al. (2019), este parâmetro pode representar o grau de maturação do fruto e indica grau de doçura do produto. 
RIBEIRO, V. H. A et al. Influência da temperatura sobre a composição físico-química de noni desidratado (Morinda citrifolia L.). In: II Congresso Paraibano de Agroecologia \& IV Exposição Tecnológica, 2019. Anais... Caderno Verde de Agroecologia e

Desenvolvimento Sustentável, Pombal, v. 9, n.7, e-7099, 2019.

Foram quantificados elevados teores de ácido ascórbico (154,2 mg/100g), valores semelhantes foram reportados por Alencar et al. (2016) em suco de noni $(166 \mathrm{mg} / 100 \mathrm{~g})$, os autores constataram que a polpa desta fruta apresenta maior teor de ácido ascórbico quando comparado com frutas como o limão, laranja e abacaxi.

O teor de cinzas apresentado na amostra foi de 0,72\%, Gazola et al. (2016) obteve valores superiores com relação a este parâmetro para polpa de pitanta $(0,90 \%)$, amora $(0,95 \%)$ e mirtilo $(0,97 \%)$.O teor de proteínas obtido no presente estudo foi de $1,15 \%$, superior ao verificado por Gazola et al. (2016) em polpa de mirtilo $(0,51 \%)$.

A polpa do noni in natura apresentou teor de lipídeos correspondente a $2,15 \%$, semelhante ao observado por Palioto et al. (2015), em nonis cultivados em Maringá-PR (2,19\%).De acordo com Palioto et al. (2015), a quantidade de proteína presente no noni representam cerca de $11,3 \%$ da sua matéria seca, compreendendo essencialmente os aminoácidos glutamina, ácido aspártico e isoleucina.

Na Tabela 2 estão expressos os resultados referentes a caracterização da polpa de noni desidratada através da secagem convectiva, utilizando as temperaturas de 50,60 e $70^{\circ} \mathrm{C}$.

Tabela 2- Caracterização físico-química da polpa de noni desidratada a 50,60 e $70{ }^{\circ} \mathrm{C}$.

\begin{tabular}{ccccc}
\hline Parâmetros & $\mathbf{5 0}^{\mathbf{}} \mathbf{C}$ & $\mathbf{6 0}^{\mathbf{}} \mathbf{C}$ & $\mathbf{7 0}^{\mathbf{}} \mathbf{C}$ & $\mathbf{C V}(\boldsymbol{\%})$ \\
\hline Umidade (\%) & $12,25^{\mathrm{a}} \pm 0,30$ & $10,42^{\mathrm{b}} \pm 0,08$ & $9,17^{\mathrm{c}} \pm 0,15$ & 2,32 \\
Atividade de água $\left(\mathrm{A}_{\mathrm{w}}\right)$ & $0,42^{\mathrm{a}} \pm 0,01$ & $0,31^{\mathrm{b}} \pm 0,01$ & $0,27^{\mathrm{c}} \pm 0,01$ & 2,70 \\
pH & $4,01^{\mathrm{a}} \pm 0,02$ & $3,71^{\mathrm{b}} \pm 0,03$ & $3,51^{\mathrm{c}} \pm 0,02$ & 0,95 \\
ATT (\% ácido cítrico) & $3,15^{\mathrm{c}} \pm 0,02$ & $2,77^{\mathrm{b}} \pm 0,03$ & $3,15^{\mathrm{a}} \pm 0,02$ & 2,90 \\
SST ( ${ }^{\mathrm{B}}$ Brix) & $50,97^{\mathrm{b}} \pm 0,24$ & $55,02^{\mathrm{a}} \pm 0,28$ & $56,36^{\mathrm{a}} \pm 0,68$ & 1,02 \\
Ratio (SST/ATT) & $20,62^{\mathrm{a}} \pm 0,42$ & $19,85^{\mathrm{a}} \pm 0,44$ & $17,88^{\mathrm{b}} \pm 0,55$ & 3,01 \\
Vitamina C (mg/kg) & $111,58^{\mathrm{c}} \pm 3,37$ & $130,84^{\mathrm{b}} \pm 1,16$ & $140,70^{\mathrm{a}} \pm 1,10$ & 2,07 \\
Cinzas (\%) & $3,30^{\mathrm{b}} \pm 0,24$ & $3,43^{\mathrm{a}} \pm 0,24$ & $3,52^{\mathrm{a}} \pm 0,24$ & 1,39 \\
Proteínas (\%) & $1,23^{\mathrm{a}} \pm 0,01$ & $1,06^{\mathrm{b}} \pm 0,06$ & $0,98^{\mathrm{b}} \pm 0,06$ & 3,19 \\
Lipídeos (\%) & $7,14^{\mathrm{b}} \pm 0,03$ & $7,30^{\mathrm{ab}} \pm 0,02$ & $7,46^{\mathrm{a}} \pm 0,03$ & 0,91 \\
\hline
\end{tabular}

Nota: Letra sobrescritas iguais na mesma linha não diferem estatisticamente entre si. Foi aplicado o Teste de Tukey ao nível de 5\% de probabilidade $(\mathrm{P}<0,05), \mathrm{CV}$ : coeficiente de variação.

Através da Tabela 2, constatou-se que o aumento da temperatura do ar de secagem proporcionou a redução do teor de umidade e de atividade de água do produto, valores inferiores destes parâmetros foram observados para a temperatura de $70{ }^{\circ} \mathrm{C}$, que apresentou teor de umidade de 9,17\% e 0,27 de atividade de água. Nunes et al. (2017) ao realizarem a secagem de resíduos de abacaxi utilizando as temperaturas de 50,60 e $70{ }^{\circ} \mathrm{C}$ verificaram comportamento semelhante, cujas umidades variaram de 9,25 a 5,41\% e houve variação de 0,56 a 0,39\% de atividade de água.

Com relação a acidez foi observada uma variação de 3,15 a 2,77\% e o pH variou de 4,01 a 3,51. Constatou-se portanto o aumento da acidez do produto devido ao acréscimo da temperatura, assim como verificado por Coelho et al. (2019) ao realizarem secagem de manga.

Foi verificado o aumento no teor de sólidos solúveis totais devido ao aumento da temperatura aplicada no processo de secagem, que variou de 50,97 a 56,36 ${ }^{\circ}$ Brix. Contudo, não foi observada diferença estatística entre as amostras secas a 60 e $70{ }^{\circ} \mathrm{C}$. Comportamento semelhante foi observado por Costa et al. (2015) ao efetuarem a desidratação de uva Itália.

Valores superiores com relação ao teor de ácido ascórbico foram verificados na amostra desidratada a $70^{\circ} \mathrm{C}$ (140,70 mg/kg), este fato é justificado pois segundo Reis et al. (2017), a perda de água nos frutos durante a secagem tendem a concentrar os nutrientes. Apesar da vitamina $C$ ser um composto termossensível, a desidratação do noni a 70 ${ }^{\circ} \mathrm{C}$ proporcionou o menor tempo do processo, reduzindo o tempo de contato do produto com temperaturas elevadas e contribuindo assim para a manutenção da qualidade nutricional do produto. Castro et al. (2019) ao realizarem a secagem de taro em temperaturas de variaram de 70 a $90{ }^{\circ} \mathrm{C}$, observaram comportamento semelhante ao verificado no presente estudo, em que o maior teor de vitamina c foi verificado na maior temperatura $(0,17 \mathrm{mg} / 100 \mathrm{~g})$ e o valor inferior $(0,05$ $\mathrm{mg} / 100 \mathrm{~g}$ ) foi observado na menor temperatura.

O maior valor com relação ao teor de proteínas foi obtido para a polpa de noni seca a $50{ }^{\circ} \mathrm{C}(1,23 \%)$, foi observada uma degradação deste componente devido ao aumento da temperatura. Silva et al. (2019) em seus estudos sobre secagem de pimentão amarelo utilizando temperaturas de 50 a $80{ }^{\circ} \mathrm{C}$, obtiveram teor de proteínas que variou de 2,15 a $1,84 \%$.

O teor lipídico variou de 7,14 a 7,46\%, sendo o valor superior obtivo na maior temperatura. Contudo, a amostra seca a $60{ }^{\circ} \mathrm{C}$ não apresentou diferença estatística significativa quando comparada aos demais tratamentos. Santos et al. (2019) ao realizarem a secagem de grãos de arroz negro nas temperaturas de 40,50,60, 70 e $80^{\circ} \mathrm{C}$ também verificaram o aumento do teor lipídico devido ao aumento da temperatura do ar de secagem. De acordo com Reis et al. (2017), a perda de água nos frutos durante a desidratação tende a concentrar os nutrientes. 
RIBEIRO, V. H. A et al. Influência da temperatura sobre a composição físico-química de noni desidratado (Morinda citrifolia L.). In: II Congresso Paraibano de Agroecologia \& IV Exposição Tecnológica, 2019. Anais... Caderno Verde de Agroecologia e

Desenvolvimento Sustentável, Pombal, v. 9, n.7, e-7099, 2019.

\section{CONCLUSÕES}

Constatou-se que o acréscimo da temperatura do ar de secagem provocou a redução do teor de umidade, atividade de água, ratio e proteínas. Porém foi observado o aumento dos seguintes parâmetros: acidez, sólidos solúveis totais, lipídeos, cinzas e vitamina c.

\section{REFERÊNCIAS}

ALENCAR, I.S.S.; VALE, B.N.; WATARO, N.O. Teor de ácido ascórbico no suco de noni e em polpas de frutas. Revista cereus, v.8, n.3, p.154-171, 2016.

BLIGH, E. G.; DYER, W. J. A rapid method of total lipid and purifi cation. Canadian Journal of Biochemistry and Physiology, v.37, n.8, p.911-917, 1959.

CASTRO, D.S.; OLIVEIRA, T.K.B.; LEMOS, D.M.; ROCHA, A.P.T.; ALMEIDA, R.D. Efeito da temperatura sobre a composição físico-química e compostos bioativos de farinha de taro obtida em leito de jorro. Brazilian Journal of Food Technology, Campinas, v. 20, p.1-5, 2017.

COELHO, B.E.; NASCIMENTO, M.M.; CARVALHO, I.R.B.; SOUSA, K.S.M.; MACHADO, N.S.; NETO, A.C. Curva de secagem solar e avaliação físico-química da manga "Espada" Solar drying curve and physical-chemical assessment of the "Sword" mango. Journal of Environmental Analysis and Progress, v.4, n.3, p.187-194, 2019.

CORDEIRO, M.H.M.; SILVA, J.M.; MIZOBUTSI, G.P.; MIZOBUTSI, E.H.; MOTA, W.F. Caracterização física, química e nutricional da pitaia-rosa de polpa vermelha. Revista Brasileira de Fruticultura, Jaboticabal, v. 37, n. 1, p. 20$26,2015$.

COSTA, J.D.S.; NETO, A.F.; NUNES, S.M.; RYBKA, A.C.P.; BIASOTO, A.C.T.; FREITAS, S.T. Caracterização física e físico-química de uva itália desidratada. Rev. Iber. Tecnología Postcosecha, v.16, n.2, p.273-280, 2015.

FIGUEIREDO, R.M.F.; GOMES, J.P.; QUEIROZ, A.J.M.; CASTRO, D.S.; LEMOS, D.M. Mathematical modeling of pequi pulp drying and effective diffusivity determination. Revista Brasileira de Engenharia Agrícola e Ambiental, Campina Grande, v.21, n.7, p.493-498, 2017.

GAZOLA, M.B.; PEGORINI, D.; LIMA, V.A.; RONCATTI, R.; TEIXEIRA, S.D.; PEREIRA, E.A. Elaboração e caracterização de bebidas à base de extrato hidrossolúvel de soja com polpa de pitanga, amora e mirtilo. B.CEPPA, Curitiba, v. 34, n. 2, p.1-14, 2016.

LIMA, A.M.S.; ARAÚJO, R.M.S.; SOUSA, J.C.S.; MEDEIROS, A.F.; SERQUIZ, R.P.; SERQUIZ, A.C.; Biotechnological Potential Assesment of Noni Fruit (Morinda citrifolialinns). Food Process Technology, v.5, n.4, p.1-6, 2017.

LIMA, I.A.S.I.; RIBEIRO, I.A.; NERIS, T.S.; SILVA, S. S.; LOSS, R.A.; GUEDES, S.F. Extração de compostos fenólicos do noni (Morinda citrifolia Linn) empregando energia ultrassônica Extraction of noni phenolic compounds (Morinda citrifolia Linn) using ultrasonic energy. Scientia Plena, v.14, n.4, p.1-7, 2018.

MORGADO, C. M.; GUARIGLIA, B. A.; TREVISAN, M. J.; FAÇANHA, R.; JACOMINO, A. P.; CORRÊA, G.; CUNHA JUNIOR, L. C. Quality assessment of jabuticabas (cv. sabará), submitted to refrigerated storage and conditioned in different packaging.Revista Interdisciplinar Da Universidade Federal Do Tocantins, v.6, n.2, p.18-25, 2019.

NUNES, J.S.; LINS, A.D.F.; GOMES, J.P.; SILVA, W.P.; SILVA, F.B. Influência da temperatura de secagem nas propriedades físico-química de resíduos abacaxi. Revista Agropecuária Técnica, Areia, v. 1, n. 1, p. 41-46, 2017.

PALIOTO, G.F.; SILVA, C.F.G.; MENDES, M.P.; ALMEIDA, V.V.; ROCHA, C.L.M.S.C.; TONIN, L.T.D. Composição centesimal, compostos bioativos e atividade antioxidante de frutos de Morinda citrifolia Linn (noni) cultivados no Paraná. Revista Brasileira de Plantas Medicinais, Campinas, v.17, n.1, p.59-66, 2015.

PIMENTEL, D.D.; MEIRA, A.M.B.; ARAÚJO, C.R.F.; PEIXOTO, M.I. O uso de noni (morinda citrofolia L.) por pacientes oncológicos: um estudo bibliográfico. Revista saúde e ciência, v.5, n.1, p.37-44, 2016. 
REIS, D.S.; NETO, A.F.; FERRAZ, A.V.; FREITAS, S.T. Produção e estabilidade de conservação de farinha de acerola desidratada em diferentes temperaturas. Brazilian Journal of Food Technology, Campinas, v. 20, p.1-7, 2017

SANDES, F.S. A.; PINTO, D.M.; CAVENAGHI, D.F.L.C.; DUARTE, J.M.A. Elaboração de frozen de noni associado com outras frutas antioxidantes, Revista eletrônica do Univag, n.12, p.1-12, 2015.

SANTOS, N.C.; BARROS, S.L.; SILVA, S.N.; RIBEIRO, V.H.A.; MELO, M.O.P.; SILVA, W.P.; ALMEIDA, R.L.J.R.; PEREIRA, T.S.; ARAÚJO, A.J.B.; GOMES, J.P.; NASCIMENTO, A.P.S.; SILVA, V.M.A.; VIEIRA, D.M. Physico-chemical characterization and kinetics of drying of organic yellow bell pepper (Capsicum annuum L.). African Journal of Agricultural Research, v.14, n.29, p.1247-1253, 2019.

SANTOS, N.C.; SILVA, W.P.; BARROS, S.L.; ARAÚJO, A.J.B.; GOMES, J.P.; ALMEIDA, R.L.J.; NASCIMENTO, A. P. S.; ALMEIDA, R.D.; SILVA, C. M. D. P. S.; QUEIROZ, A. J. M.; FIGUEIREDO, R. M. F. Study on Drying of Black Rice (Oryza sativa L.) Grains: Physical-Chemical and Bioactive Quality. Journal of Agricultural Science, v.11, n. 9, 2019.

SILVA, F. A. S; AZEVEDO, C. A. V. The Assistat Software Version 7.7 and its use in the analysis of experimental data. African Journal of Agricultural Research, v.11, n.39, p.3733-3740, 2016.

SILVA, S.N.; SILVA, P.B.; SILVA, R.M.; SILVA, L.P.F.R.; BARROSO, A.J.R.; ALMEIDA, F.A.C.; GOMES, J.P. Composição físico-química e colorimétrica da polpa de frutos verdes e maduros de Cereus jamacaru. Magistra, Cruz das Almas, v.30, p. 11-17, 2019.

SOUSA, S.F.; QUEIROZ, A.J.M.; FIGUEIRÊDO, R.M.F.; SILVA, F.B. Comportamento reológico das polpas de noni integral e concentradas. Brazilian Journal of Food Technology, Campinas, v. 20, p.1-10, 2017.

\section{AGRADECIMENTOS}

Apoio financeiro: Fundação de Apoio à Pesquisa do Estado da Paraíba - FAPESQ. 